\title{
Serum lipids and apolipoproteins in children with Type 1 (insulin-dependent) diabetes during the first two years of the disease
}

\author{
M. Kobbah, B. Vessby and T. Tuvemo \\ Departments of Paediatrics and Geriatrics, University of Uppsala, Uppsala, Sweden
}

\begin{abstract}
Summary. Serum lipoproteins and apolipoproteins were studied at diagnosis and 6,12 and 24 months later in 30 consecutive children aged 3-15 years with newly detected Type 1 (insulin-dependent) diabetes mellitus (December 1982-October 1984) and in 44 healthy control children. Serum triglycerides at diagnosis were significantly higher than after 6-24 months and also higher than in the control group ( $p<$ 0.001 ). At follow-up, triglycerides in the very low density lipoproteins and low density lipoproteins were restored to normal, while high density lipoprotein triglycerides remained high. Serum cholesterol at onset of diabetes was significantly higher than in the control children $(p<0.01)$, mainly because of increased very low density lipoprotein cholesterol $(p<$ 0.001). Cholesterol in serum and in the serum lipoprotein fractions was similar to that in the control children at followup, except that high density lipoprotein cholesterol was higher in the diabetic children after 6 months. The concentrations of the serum apolipoproteins A-I, A-II and B were higher at onset of diabetes than in the control children $(p<0.001, p<$ $0.01, p<0.05$ respectively), with a significantly increased ratio of apolipoprotein A-I to A-II in the diabetic children
\end{abstract}

$(p<0.001)$. The serum apolipoprotein concentrations were normalised during treatment. The ratio of apolipoprotein A-I to B did not differ from that in control children. On admission, there were strong positive correlations between $\mathrm{HbA}_{1 \mathrm{c}}$ and the concentrations of the very low density lipoproteins and the low density and high density lipoprotein triglycerides. There were also significantly positive correlations $(p<$ 0.01 ) between $\mathrm{HbA}_{1 \mathrm{c}}$ and apolipoprotein A-I and apolipoprotein $B$ respectively. After treatment these correlations disappeared, except for a positive correlation with very low density lipoprotein triglycerides at 2 years. In conclusion, at diagnosis, when the diabetic children were in an insulin-deficient state, all apolipoproteins and serum lipoprotein fractions, except cholesterol in high density lipoproteins and low density lipoproteins were increased. During the first two years of treatment the concentrations of lipoproteins and apolipoproteins in serum are similar to those in healthy children.

Key words: Lipoproteins, apolipoproteins, triglycerides, cholesterol, Type 1 (insulin-dependent) diabetes mellitus.
Accelerated atherosclerosis is a major cause of early death in Type 1 (insulin-dependent) diabetes mellitus. The excessive occurrence of cardiovascular disease in diabetes mellitus can be explained at least partly by increased levels of general risk factors for coronary heart disease [1]. As serum lipids are highly correlated to the development of atherosclerosis in non-diabetic subjects, it has been considered important to investigate the concentrations of lipoproteins and apolipoproteins in diabetic patients in an attempt to clarify the underlying mechanisms.

Studies on different serum lipids in children with Type 1 diabetes have so far yielded somewhat confusing results. In some studies the concentration of high density lipoprotein cholesterol (HDL Chol) was found to be higher than in healthy persons $[2,3]$, whereas in another study it was lower, especially during poor diabetes control [4]. Apolipoprotein (apo) A-I was increased in diabetic children in one of our earlier investigations [5]. It has been suggested that the high-density lipoproteins in diabetic children may be qualitatively different from those in healthy control children [3]. We also observed that the plasma lipid ester fatty acid pattern was slightly different in these patients than in healthy control children, the content of linoleic acid being higher [6]. Considered together, these data do not clearly indicate that Type 1 diabetes in young persons is associated with lipoprotein abnormalities which would predispose for development of atherosclerotic cardiovascular disease in the future. All the studies mentioned were performed in patients who had had insulin-treated diabetes for quite a few years. To the 
best of our knowledge there have been no reports on studies of the very early changes in serum lipoproteins in diabetic children.

In the present study the concentrations of lipoprotein lipid and apolipoproteins in serum during the first two years of diabetes were investigated with the aim of elucidating possible aberrations of lipoprotein metabolism that in the long term might predispose for macrovascular angiopathy.

\section{Subjects and methods}

The study group comprised 30 children aged 3-15 years referred to the University Hospital, Uppsala, with newly diagnosed diabetes mellitus, who were followed up for at least 2 years. The children represented all children with recent diabetes mellitus in the County of Uppsala in this age group during the study period. Nineteen of the 30 children were girls and 11 boys. Most of the children were prepubertal, both diabetic patients and control subjects. None of diabetic girls had a history of menarche on admission or during the two years of follow-up, except one. Very few, if any, of the diabetic boys reached puberty during the study. The diabetic children were studied immediately after diagnosis before the first insulin injection (day 0) and 6 months, one year and two years later. Fourty-four healthy children aged 4-16 years, 17 boys and 27 girls, served as control subjects. Twenty-four of the control children were peers of diabetic children in the study group, matched well for sex and age. Twenty control children were relatives or neighbours of hospital staff representing a similar age group. The diabetic children were treated with intravenous insulin infusion for the first $48-72 \mathrm{~h}$, and then with two doses of combinations of regular (Actrapid, Novo, Copenhagen, Denmark - 21 patients December 1982-April 1984; Humulin regular, Eli Lilly, Indianapolis, Ind, USA - 9 patients April 1984-October 1984) and intermediate insulins (Monotard in the former group and Humulin NPH in the latter group). During the second year of the study some of the patients were given three daily injections of insulin after the remission period.

Blood samples were taken at $7.00-8.00$ hours in the fasting state and before the morning insulin injection in all cases except on day 0 in the diabetic children. In those children, blood samples were taken immediately after the diagnosis of diabetes and before the patient had received the very first insulin injection. Fasting plasma glucose and urine glucose were determined by routine methods. Haemoglobin $(\mathrm{Hb}) \mathrm{A}_{1}$ was measured up to March 1984 and $\mathrm{A}_{1 \mathrm{c}}$ from April 1984-April 1985 by standard microcolumn chromatography (Biorad, Richmond, Calif, USA) and $\mathrm{HbA}_{\text {ic }}$ was later measured by high pressure liquid chromatography (FPLC, Pharmacia, Uppsala, Sweden). All values are expressed as $\mathrm{HbA}_{\mathrm{tc}}$.

\section{Lipoprotein separation}

Lipoprotein lipid concentrations in serum were determined. Very low density lipoproteins (VLDL), low density lipoproteins (LDL) and HDL were isolated with a combination of preparative ultracentrifugation [7] and precipitation with a sodium phosphotungstate and magnesium chloride solution [8]. Triglyceride (TG) and cholesterol (Chol) concentrations were measured in serum and in the isolated lipoprotein fractions by enzymatic methods, using BoehringerMannheim (Munich, FRG) kits 126012 and 124087, modified for use in a Multistat III F/LS apparatus (Instrumentation Laboratories, Lexington, Mass, USA). The concentrations of serum apo B, A-I and A-II were determined by turbidimetry in the Multistat III F/LS apparatus, using monospecific polyclonal antibodies against apo B, A-I and A-II respectively. Before assay the samples were pre-incubated with triglyceride lipase as suggested by $\mathrm{DaCol}$ and Kostner [9].

\section{Statistical analysis}

In the comparisons between patients and control children, Student's t-test was used on normally distributed data and Wilcoxon's signed rank test on data with skewed distributions (all triglyceride data and VLDL cholesterol). The changes in the diabetic group were studied by analysis of variance. In the comparisons between diabetic children and healthy control subjects, separate analyses were also performed in which sex and age were taken into account $(<10$ years, $\geq$ 10 years). Correlations within a group were tested by Spearman's coefficient of correlation $\left(\mathrm{r}_{\mathrm{s}}\right)$.

\section{Results}

\section{Healthy control children}

The two reference groups did not differ from each other with regard to lipoprotein or apolipoprotein concentrations, except for a small difference in LDL triglycerides. This difference did not influence the conclusions drawn from the results presented. The two control populations $(n=24 ; n=20)$ were therefore pooled in the further analyses. In this combined control group there was no significant difference between boys and girls or between younger and older children $(<$ 10 years, $\geq 10$ years) in any of the 11 studied variables.

\section{Diabetic children}

On admission, the relative body weight of the diabetic children was lower than that of the matched healthy control children (the peers), and the subscapular skin fold thicknesses in these two groups were $6.7 \pm 2.0$ and $8.5 \pm 2.5 \mathrm{~mm}$ respectively. However, all children had a body weight within the normal range (mean $\pm 2 S D$ ) for Swedish children of corresponding age. During treatment, the body weight of the diabetic children increased and at 2 years the relative body weight was similar to that of the healthy control children. Interestingly, at this time, while the subscapular skin fold thicknesses in diabetic and healthy boys were the same, the diabetic girls had a significantly greater skin fold thickness than their healthy counterparts, in spite of the similarity in relative body weight $(p<0.05)$.

The mean serum TG concentration in the diabetic children was significantly higher at the time of diagnosis of the diabetes than at the three follow-ups, and was also higher at diagnosis than in the control group $(p<0.001)$ (Table 1). These differences were mainly due to greatly increased concentrations of TG in the VLDL fraction $(p<0.001)$. LDL TG and HDL TG were also significantly higher at diagnosis $(p<0.001)$ than in the control group. During the first year of the follow-up period HDL TG remained slightly but significantly elevated in the diabetic group, while VLDL TG was transiently somewhat lower than in the control children after six months. After 2 years, however, neither of these two variables differed between the two groups. 
Table 1. Lipoprotein concentrations in whole serum and in serum density fractions (mmol/l) in diabetic children at diagnosis and 6 , 12 and 24 months later, and corresponding values for healthy control children. Significant differences from healthy control children: ${ }^{\mathrm{a}} p<0.05$; ${ }^{\mathrm{b}} p<0.01 ;{ }^{\mathrm{c}} p<0.001$. TG $=$ triglycerides. Chol $=$ cholesterol

\begin{tabular}{|c|c|c|c|c|c|c|}
\hline \multirow[t]{3}{*}{ Mean \pm SD } & \multicolumn{2}{|c|}{ Control children $(n=44)$} & \multicolumn{4}{|c|}{ Diabetic children $(n=30)$} \\
\hline & & & \multicolumn{4}{|c|}{ Time (months) } \\
\hline & & & 0 & 6 & 12 & 24 \\
\hline \multirow[t]{2}{*}{ VLDL } & TG & $0.36 \pm 0.27$ & $2.05 \pm 2.47^{\mathrm{c}}$ & $0.22 \pm 0.15^{b}$ & $0.25 \pm 0.15$ & $0.29 \pm 0.16$ \\
\hline & Chol & $0.15 \pm 0.10$ & $0.65 \pm 0.68^{c}$ & $0.12 \pm 0.08$ & $0.12 \pm 0.07$ & $0.12 \pm 0.08$ \\
\hline \multirow[t]{2}{*}{ LDL } & TG & $0.32 \pm 0.11$ & $0.53 \pm 0.21^{\mathrm{c}}$ & $0.27 \pm 0.08$ & $0.30 \pm 0.09$ & $0.31 \pm 0.06$ \\
\hline & Chol & $3.01 \pm 0.63$ & $3.11 \pm 0.60$ & $2.99 \pm 0.80$ & $3.08 \pm 0.88$ & $2.94 \pm 0.70$ \\
\hline \multirow[t]{2}{*}{ HDL } & TG & $0.15 \pm 0.04$ & $0.33 \pm 0.12^{\mathrm{c}}$ & $0.21 \pm 0.07^{c}$ & $0.19 \pm 0.05^{b}$ & $0.18 \pm 0.06$ \\
\hline & Chol & $1.11 \pm 0.19$ & $1.14 \pm 0.30$ & $1.21 \pm 0.20^{\mathrm{a}}$ & $1.13 \pm 0.26$ & $1.18 \pm 0.20$ \\
\hline \multirow[t]{2}{*}{ Whole serum } & TG & $0.87 \pm 0.34$ & $2.88 \pm 2.59^{c}$ & $0.75 \pm 0.21$ & $0.79 \pm 0.21$ & $0.85 \pm 0.22$ \\
\hline & Chol & $4.33 \pm 0.66$ & $4.84 \pm 0.85^{a}$ & $4.35 \pm 0.85$ & $4.41 \pm 0.89$ & $4.29 \pm 0.80$ \\
\hline $\mathrm{HbA}_{1 \mathrm{c}}(\%)$ & & $4.9 \pm 0.5$ & $10.4 \pm 2.1^{\mathrm{c}}$ & $6.2 \pm 1.4^{c}$ & $7.0 \pm 1.3^{c}$ & $7.8 \pm 2.0^{c}$ \\
\hline $\begin{array}{l}\text { Plasma glucose } \\
(\mathrm{mmol} / 1)\end{array}$ & & $4.5 \pm 0.3$ & $19.6 \pm 8.3^{\mathrm{c}}$ & $6.9 \pm 3.5^{\mathrm{b}}$ & $7.0 \pm 2.9^{c}$ & $10.6 \pm 5.3^{c}$ \\
\hline
\end{tabular}

Table 2. Serum apolipoprotein concentrations (mg/dl) in the children with Type 1 (insulin-dependent) diabetes during the first two years of the disease and in healthy control children. Significant differences from healthy control children: ${ }^{\mathrm{a}} p<0.05 ;{ }^{\mathrm{b}} p<0.01 ;{ }^{\mathrm{c}} p<0.001$

\begin{tabular}{|c|c|c|c|c|c|c|}
\hline \multirow[t]{3}{*}{ Mean $\pm S D$} & \multicolumn{2}{|c|}{ Control children $(n=44)$} & \multicolumn{4}{|c|}{ Diabetic children $(n=30)$} \\
\hline & & & \multicolumn{4}{|l|}{ Time (months) } \\
\hline & & & 0 & 6 & 12 & 24 \\
\hline \multirow[t]{5}{*}{ Apolipoprotein } & A-I & $114.9 \pm 13.5$ & $141.5 \pm 26.3^{c}$ & $118.0 \pm 14.8$ & $120.6 \pm 13.4$ & $118.5 \pm 17.6$ \\
\hline & A-II & $41.5 \pm 4.2$ & $45.0 \pm 6.2^{\mathrm{a}}$ & $41.4 \pm 6.8$ & $41.8 \pm 5.7$ & $41.0 \pm 5.2$ \\
\hline & B & $66.4 \pm 14.1$ & $76.0 \pm 15.7^{\mathrm{a}}$ & $63.9 \pm 18.4$ & $64.8 \pm 17.1$ & $65.0 \pm 12.5$ \\
\hline & A-I/A-II & $2.78 \pm 0.27$ & $3.15 \pm 0.41^{c}$ & $2.88 \pm 0.28$ & $2.91 \pm 0.31$ & $2.89 \pm 0.29$ \\
\hline & $\mathrm{A}-\mathrm{I} / \mathrm{B}$ & $1.79 \pm 0.34$ & $1.90 \pm 0.36$ & $1.96 \pm 0.51$ & $1.96 \pm 0.47$ & $1.88 \pm 0.41$ \\
\hline
\end{tabular}

The mean serum cholesterol concentration was significantly higher at diagnosis in the children with diabetes mellitus than in the control group $(p<0.05)$. This difference was due to high levels of VLDL cholesterol, which were significantly increased at that time $(p<$ 0.001). The LDL and HDL cholesterol fractions were not significantly changed at onset of diabetes. After 6 months HDL Chol was increased in the diabetic children, and it was also slightly higher at 2 years, although it was not statistically different at that time from the value in the control children. With this exception, the cholesterol concentrations in all serum fractions and in whole serum were similar to those of the control children during the follow-up period.

The mean apo A-I concentration was increased at diagnosis in the diabetic children in comparison with healthy control children $(p<0.001)$ (Table 2). After six months to two years the level remained stable and did not differ from the value in the control group.

The concentrations of apo A-II and apo B behaved like that of A-I, with increased initial values which were restored to normal as early as at six months, but the changes were of lower significance from those in the control children (A-II, $p<0.05 ; \mathrm{B}, p<0.05$ ).

The ratio of apo A-I to A-II was significantly higher in the diabetic patients on admission than in the control children $(p<0.001)$. After institution of insulin, the ratio was restored to normal and did not differ significantly from that in the control group. The ratio of apo A-I to apo B did not differ from that in the control subjects.

\section{Relationships between lipoprotein variables and degree of carbohydrate control}

On admission there were strong positive correlations between the $\mathrm{HbA}_{1 \mathrm{c}}$ concentration and the concentrations of VLDL TG $(r=0.63 ; p<0.001)$, VLDL Chol $(r=0.62 ; p<0.001), \mathrm{LDL}$ TG $(r=0.41 ; p<0.05)$, HDL TG $(r=0.65 ; p<0.01)$, serum TG $(r=0.72 ; p<0.001)$ and serum Chol $(r=0.53 ; p<0.01)$ respectively. Similar, but weaker correlations were found between these lipoproteins and plasma glucose. $\mathrm{HbA}_{1 \mathrm{c}}$ was also significantly correlated to both apo $\mathrm{B}(r=0.52 ; p<0.01)$ and apo A-I $(r=0.59 ; p<0.01)$.

After insulin treatment most of the correlations between the variables of carbohydrate control and the lipoproteins disappeared. At 2 years the concentration of $\mathrm{HbA}_{1 \mathrm{c}}$ was positively correlated with VLDL TG $(r=0.49 ; p<0.01)$ and serum TG $(r=0.42 ; p<0.05)$, respectively, but no longer showed any significant correlation with the serum apolipoprotein concentra- 
tions. Neither at six months, one year nor two years were there any significant correlations between the insulin dosage per kilogram body weight and the lipoprotein variables tested.

\section{Relationships between apolipoprotein and lipoprotein lipid concentrations}

On admission apo A-I showed significant linear correlations with the concentrations of VLDL cholesterol $(r=0.52 ; p<0.01)$, LDL triglycerides $(r=0.54 ; p<$ $0.01)$ and HDL triglycerides $(r=0.59 ; p<0.01)$. The correlation between apo A-I and HDL Chol was also positive but weaker $(r=0.48 ; p<0.05)$. Apo A-I displayed a strong positive correlation with serum cholesterol $(r=0.63 ; p<0.001)$ and a significant correlation with apo B $(r=0.49 ; p<0.05)$, but no correlation with LDL Chol. Apo B was significantly correlated to VLDL TG $(r=0.42 ; p<0.05)$, VLDL Chol $(r=0.45$; $p<0.05)$, LDL TG $(r=0.58 ; p<0.01)$ and HDL TG $(r=0.43 ; p<0.05)$, and also with LDL Chol $(r=0.69$; $p<0.001)$.

After two years of insulin treatment no significant correlations remained between apo A-I or apo B and the concentrations of VLDL, LDL triglycerides or HDL triglycerides. There was no significant correlation between apo A-I and apo B. On the other hand, at this time, when all the apolipoprotein and lipoprotein concentrations were virtually normal, there was a very strong positive correlation between apo A-I and HDL cholesterol $(r=0.90 ; p<0.001)$, indicating that the major variation of apo A-I was now related to the HDL concentration. Similarly, apo B was strongly correlated only to LDL Chol $(r=0.90 ; p<0.001)$ at this time.

\section{Influence of age and sex}

At onset of diabetes mellitus the values for nine of the 11 variables analysed differed from the corresponding values in the healthy control children. The differences between diabetic children and control subjects could not be explained by differences in a specific sex or age group, the pattern being similar in all the subgroups analysed.

\section{Discussion}

In earlier studies we found that in children who had had diabetes for more than two years, treated in the same way as the patients of this study, the HDL cholesterol [2] and apo A-I concentrations were increased [5]. The group of homogeneously treated children with diabetes in the present study was found to have concentrations of lipoproteins and apolipoproteins in whole serum similar to those in healthy children during the first two years of treatment, except for significantly increased HDL TG the first year and HDL chol at six months. However, at the time of diagnosis all apolipoproteins and the serum lipoprotein triglyceride and cholesterol concentrations, except cholesterol in HDL and LDL, were significantly increased.

The blood sampling on admission differed from that performed later by not being systematically done in the fasting state. Children referred to hospital for overt diabetes are in a catabolic condition, most often with a poor appetite. At the time of blood sampling they had been under investigation for some hours, with no opportunity to have any food. Differences in their lipoprotein pattern, as compared with that in the control children, would therefore be expected not to be due to non-fasting but rather to be the effect of the disease state. Although it cannot be excluded that dietary factors may have contributed to the triglyceride increase in a few patients, it is not plausible that this could explain the rather pronounced lipoprotein aberrations seen in every one of these children as compared with healthy children on average Swedish diets.

The simultaneous elevation of the VLDL lipid levels and the LDL and HDL triglycerides is a common pattern in hypertriglyceridaemic syndromes. The high VLDL lipid levels are due to accumulation of triglyceride-rich particles in the circulation. The pronounced increase in LDL triglycerides, in combination with normal LDL cholesterol, indicates an increased content of intermediary lipoproteins with a high triglyceride content (density <1.019) within the conventional LDL density range (1.006-1.063). The reason for the increased HDL triglycerides is probably an exchange of cholesterol esters for triglycerides between HDL and VLDL and LDL, mediated by the cholesterol ester transfer protein [10].

The hypertriglyceridaemia on admission may have been due to a combination of increased hepatic triglyceride production and delayed removal of triglyceriderich lipoproteins [11]. The insulin deficiency results in enhanced lipolysis, leading to a high concentration of circulating free fatty acids, which together with the high blood glucose concentration may serve as precursors for triglyceride synthesis. On the other hand, the capacity for hepatic triglyceride synthesis is limited, owing to the lack of insulin, favouring oxidation of the fatty acids to ketone bodies.

Lipoprotein lipase, the key enzyme in the hydrolysis of circulating lipoprotein triglycerides, is activated by insulin $[12,13]$. In an insulin-deficient state the lipoprotein lipase activity decreases, causing accumulation of lipoprotein triglycerides in the circulation [14]. This may be the major cause of the fairly pronounced hypertriglyceridaemia in some of the diabetic children (Fig. 1).

After commencement of insulin treatment, the activity of lipoprotein lipase becomes normalised, lipolysis is reduced and the glucose concentration is lowered. The slightly subnormal VLDL triglyceride concentration, associated with an increased HDL choles- 


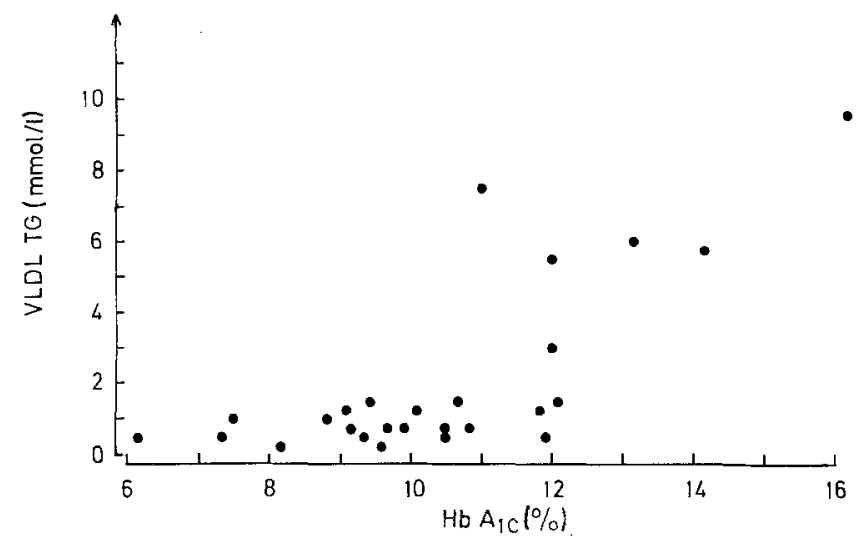

Fig. 1. Relation betwen $\mathrm{HbA}_{1 \mathrm{c}}$ and the triglyceride concentration in very low density lipoprotein triglycerides (VLDL TG) in children with diabetes mellitus on admission $(r=0.63, p<0.001)$

terol concentration at six months, may be explained by relatively high peripheral insulin concentrations [15], leading to higher than normal lipoprotein lipase activity. It has been shown repeatedly that HDL cholesterol increases during insulin treatment [16-19] and evidence has been presented to indicate that this increase may be due to increased lipoprotein lipase activity [11]. Whether the normal HDL cholesterol on admission, in spite of insulin deficiency with low lipoprotein lipase activity and high serum triglycerides, may have been due to low activity of hepatic lipase also [20,21], or to changes in the properties of the apolipoproteins caused by glycosylation [22], or both, is a matter of speculation.

An interesting finding in this study was that the concentrations of apo A-I, A-II and B were increased on admission, in spite of normal concentrations of HDL and LDL cholesterol; but were restored to normal during insulin treatment. The relative increases in apo A-I $(+16 \%)$ and apo B $(+14 \%)$ were more pronounced than that in apo A-II $(+11 \%)$. As a consequence, the mean ratio of apo A-I to apo A-II was significantly higher $(p<0.001)$ on admission than that in the control subjects, but returned to normal during treatment. This may indicate that not only the concentration but also the composition of HDL is abnormal in insulin-deficient diabetes. This was also suggested by Eckel et al. [23], who found increased A-I/A-II ratios in insulin-treated diabetes. However, an alternative explanation for the relatively high levels of apo A-I and apo B in our insulin-deficient diabetic children is that they are due to the presence of the former apolipoproteins in triglyceride-rich lipoproteins with a density of $<1.019$. Compatible with this concept was the finding that aop A-I and aop B were normalised (decreased) during treatment, in parallel with the concentrations of the very low density lipoproteins and the LDL triglycerides, while the LDL and HDL cholesterol levels remained unchanged or increased slightly. Also, the finding in the present study of strong correla- tions of both apo A-I and apo B with the concentrations of the VLDL cholesterol and LDL triglycerides on admission, is compatible with the view that a significant proportion of these apolipoproteins was transported in lipoproteins with a density of $<1.019$. In normal subjects there is an inverse correlation between apo A-I and the plasma triglycerides [24] and the concentrations of apo A-I (and HDL cholesterol) and apo $B$ tend to vary inversely.

A significant proportion of apo A-I is synthesized in the intestine and incorporated into the chylomicrons $[25,26]$. Subsequent to hydrolysis of the chylomicron triglycerides by lipoprotein lipase, surface material containing apo A-I is transferred to HDL [26]. As a consequence of delayed chylomicron hydrolysis due to insulin deficiency, an increased proportion of apo A-I in serum may remain in the lipoproteins and with density $<1.006$. Simultaneously, the content of apo B will increase in lipoproteins with a density of $<1.019$, as a result of retarded removal of chylomicrons, VLDL and lipoproteins of intermediate density. The increased ratio A-I/A-II is compatible with the fact that the intestine seems to make a less important contribution to apo A-II synthesis than to apo A-I synthesis [27]. In experimental studies on diabetic rats [28], apo A-I, A-IV and $\mathrm{B}$ were found to be increased in lipoproteins with a density of $<1.006$, while the content of apo $E$ was reduced. In a subsample of the diabetic patients the apolipoprotein concentrations in the lipoprotein fraction with density $>1.006$ was determined both on admission and at one year. The apolipoprotein concentrations in the lipoproteins with density $<1.006$ was estimated as the difference between the concentrations in whole serum and in the lipoprotein fraction with density $>1.006$. In all patients a higher proportion of both apolipoprotein B and A-I was found in lipoproteins with density $<1.006$ on admission than at 1 year (unpublished data).

In accordance with the above discussion, an increased apo A-I concentration in diabetic patients may not be indicative of an "anti-atherosclerotic" state, but rather of relative insulin deficiency and delayed triglyceride removal. Apo B has been considered a sensitive indicator of glycaemic control in adult diabetic patients [29], with reduced levels during intensified insulin treatment. The relationships between $\mathrm{HbA}_{1 \mathrm{c}}$ and the lipoprotein composition on admission indicate that the relative increases of both apo B and A-I were related to glycaemia; the higher the concentrations of apolipoproteins, the poorer the metabolic control.

Whether an increased HDL concentration, if due to a high peripheral insulin concentration and enhanced lipoprotein lipase activity, is beneficial remains an open question.

Finally this study also shows that it is possible to achieve a virtually normal lipoprotein pattern in diabetic children during a period of intensive treatment with a dietary regimen and insulin. 
Acknowledgements. This investigation was supported by grants from the "Förenade Liv" Mutual Group Life Insurance Company, Stockholm, Sweden, the Nordic Insulin Foundation and the Swedish Diabetic Association.

\section{References}

1. Pyörälä K, Laakso M (1983) Macrovascular disease in diabetes mellitus. In: Mann JI, Pyörälä K, Teusscher A (eds) Diabetes in epidemiological perspective. Churchill Livingstone, Edinburgh London Melbourne New York, pp 183-247

2. Ewald U, Gustafson S, Tuvemo T, Vessby B (1984) Increased high density lipoproteins in diabetic children. Eur J Pediatr 142: 154-156

3. Klujber L, Molnar D, Kardos M, Jaszai V, Soltecz G, Mestyan J (1979) Metabolic control, glycosylated haemoglobin and high density lipoprotein cholesterol in diabetic children. Eur J Pediatr 132: 284-297

4. Lopes-Virella MF, Wohltmann JH, Loadholt CB, Buse MG (1981) Plasma lipids and lipoproteins in young insulin-dependent diabetic patients: relationship with control. Diabetologia 21: 216-223

5. Ewald U, Tuvemo T, Vessby B, Wålinder O (1982) Serum apolipoproteins A-I, A-II and B in diabetic children and matched healthy controls. Acta Paediatr Scand 71: 15-18

6. Ewald U, Gustafsson I-B, Tuvemo T, Vessby B (1982) Fatty acid composition of serum lipids in diabetic children and their matched healthy controls. Ups J Med Sci 87: 111-117

7. Havel RI, Eder HA, Bragdon JH (1955) The determination and chemical composition of ultracentrifugally separated lipoproteins in human serum. J Clin Invest 34: 1345-1353

8. Seigler L, Wu WT (1981) Separation of serum high-density lipoprotein for cholesterol determination: ultracentrifugation vs precipitation with sodium phosphotungstate and magnesium chloride. Clin Chem 27: 838-841

9. DaCol P, Kostner GM (1983) Immuniquantification of total apolipoprotein B in serum by nephelometry: influence of lipase treatment and detergents. Clin Chem 29: 1045-1050

10. Barter PJ, Ha YG, Calvert GB (1981) Studies of esterified cholesterol in subfractions of plasma high-density lipoproteins. Atherosclerosis 38: 165-175

11. Nikkilä EA, Kekki M (1973) Plasma triglyceride transport kinetics in diabetes mellitus. Metabolism 22: 1-22

12. Taskinen MR, Nikkilä EA (1979) Lipoprotein lipid activity of adipose tissue and skeletal muscle in insulin-deficient human diabetics. Diabetologia 17: 351-356

13. Pfeifer MA, Brunzell JD, Best JD, Judzewitsch RG, Halter JB, Porte D Jr (1983) The response of plasma triglyceride, cholesterol and lipoprotein lipase to treatment in non-insulin-dependent diabetic subjects without familial hypertriglyceridemia. Diabetes 32 : 525-531

14. Chait A, Robertson TH, Brunzell JD (1981) Chylomicronemia syndrome in diabetes mellitus. Diabetes Care 4: 343-348
15. Nikkilä EA, Hormila $P(1978)$ Serum lipids and lipoprotein in insulin treated diabetes. Demonstration of increased high-density lipoprotein concentrations. Diabetes 27: 1078-1086

16. Nikkilä EA (1981) High density lipoproteins in diabetes. Diabetes 30 [Suppl 2]: 82-87

17. Dunn FL, Pietri A, Raskin P (1981) Plasma lipid and lipoprotein levels with continuous subcutaneous insulin infusion in type I diabetes mellitus. Ann Intern Med 95: 426-431

18. Falko JM, O'Dorisio TM, Lataland S (1982) Improvement of high-density lipoprotein-cholesterol levels. J Am Med Assoc 247: 37-39

19. Lawson P, Trayner I, Rosenstock J, Kohner E (1984) The effect of continuous subcutaneous insulin infusion on serum lipids. Diabete Metabol (Paris) 10: 239-244

20. Harno K, Nikkilä EA, Kuusi T (1980) Plasma HDL-cholesterol and post-heparin plasma hepatic endothelial lipase (HL) activity: relationship to obesity and non-insulin dependent diabetes (NIDDM). Diabetologia 19: 281

21. Abrams JJ, Ginsberg H, Grundy SM (1982) Metabolism of cholesterol and plasma triglycerides in non-ketotic diabetes mellitus. Diabetes 31: 903-910

22. Kennedy L, Baynes JW (1984) Non-enzymatic glycosylation and the chronic complications of diabetes: an overview. Diabetologia 26: 93-98

23. Eckel RH, Albers JJ, Cheung MC, Wahl PW, Lindgren FT, Bierman EL (1981) High density lipoprotein composition in insulindependent diabetes mellitus. Diabetes 30: 132-138

24. Schafer EJ, Zech LA, Jenkins LL, Bronzert TJ, Rubalcaba EA, Lindgren FT, Aamodt RL, Brewer HB (1982) Human apolipoprotein A-I and A-II metabolism. J Lipid Res 23: 850-862

25. Nicoll A, Miller NE, Lewis B (1980) High density lipoprotein metabolism. Adv Lipid Res 17: 53-106

26. Tall AR, Small DM (1978) Plasma high density lipoproteins. N Engl J Med 299: 1232-1236

27. Green PHR, Glickman RM, Saudek CD, Blum CB, Tall AR (1979). Human intestinal lipoproteins. Studies in chyluric subjects. J Clin Invest 64: 233-242

28. O'Looney P, Irwin D, Briscoe P, Vahouny GV (1985) Lipoprotein composition as a component in the lipoprotein clearance defect in experimental diabetes. J Biol Chem 260: 428-432

29. Gonen B, White N, Schonfeld G, Skor D, Miller P, Santiago J (1985) Plasma levels of apoprotein B in patients with diabetes mellitus: the effect of glycaemic control. Metabolism 34: 675-679

Received: 21 September 1987

and in revised form: 10 February 1988

Dr. T. Tuvemo

Department of Paediatrics

Akademiska Hospital

S-751 85 Uppsala

Sweden 